\title{
Positive solution for singular third-order BVPs on the half line with first-order derivative dependence
}

\author{
Abdelhamid Benmezaï \\ Faculty of Mathematics, USTHB, \\ Algiers, Algeria \\ email: aehbenmezai@gmail.com
}

\author{
El-Djouher Sedkaoui \\ Faculty of Mathematics, USTHB, \\ Algiers, Algeria \\ email: sedkaouisedkaoui@gmail.com
}

Abstract. In this paper, we investigate the existence of a positive solution to the third-order boundary value problem

$$
\left\{\begin{array}{l}
-u^{\prime \prime \prime}(t)+k^{2} u^{\prime}(t)=\phi(t) f\left(t, u(t), u^{\prime}(t)\right), t>0 \\
u(0)=u^{\prime}(0)=u^{\prime}(+\infty)=0
\end{array}\right.
$$

where $k$ is a positive constant, $\phi \in \mathrm{L}^{1}(0,+\infty)$ is nonnegative and does vanish identically on $(0,+\infty)$ and the function $f: \mathbb{R}^{+} \times(0,+\infty) \times$ $(0,+\infty) \rightarrow \mathbb{R}^{+}$is continuous and may be singular at the space variable and at its derivative.

\section{Introduction and main results}

Boundary value problems for third-order differential equations arise in many branches of physics and engineering where, for physical considerations, the positivity of the solution is required. For instance, Danziger and Elemergreen (see [15], p. 133) have obtained the following third-order linear differential equations: 


$$
\begin{aligned}
& \alpha_{3} u^{\prime \prime \prime}+\alpha_{2} u^{\prime \prime}+\alpha_{1} u^{\prime}+(1+k) u=k c, \theta<c \text { and } \\
& \alpha_{3} u^{\prime \prime \prime}+\alpha_{2} u^{\prime \prime}+\alpha_{1} u^{\prime}+u=0, \theta>c .
\end{aligned}
$$

These equations describe the variation of thyroid hormone with time. Here $u=u(t)$ is the concentration of thyroid hormone at time $t$ and $\alpha_{3}, \alpha_{2}, \alpha_{2}, k$ and $\mathrm{c}$ are constants.

A reduced version of the Hodgkin-Huxley model was proposed by Nagumo. He suggested the class of third-order differential equation

$$
u^{\prime \prime \prime}-c u^{\prime \prime}+f^{\prime}(u) u^{\prime}-\frac{b}{c} u=0
$$

as a model exhibiting many of the features of the Hodgkin-Huxley equations, where $f$ is a regular function. The Hodgkin-Huxley model is a system of nonlinear differential equations that approximates the electrical characteristics of excitable cells such as neurons and cardiac myocytes. Recall that the HodgkinHuxley model describes the ionic mechanisms underlying the initiation and propagation of action potentials in the squid giant axom. The model has played a vital role in biophysics and neuronal modelling. For more details of Nagumo's equations, we refer to the paper by McKeen [22].

The Kuramoto-Sivashinsky equation

$$
u_{t}+u_{x x x x}+u_{x x}+\frac{1}{2} u^{2}=0
$$

arises in a wide variety of physical phenomena. It was introduced to describe pattern formulation in reaction diffusion systems, and to model the instability of flame front propagation (see Y. Kuramoto and T. Yamada [18] and D. Michelson [23]). The travelling wave solutions of this partial differential equation (i.e. $\mathfrak{u}(x, t)=\mathfrak{u}(x-c t))$ solve the nonlinear third-order differential equation

$$
\lambda u^{\prime \prime \prime}(x)+u^{\prime}(x)+f(u)=0,
$$

where $\lambda$ is a parameter depend on the constant $c$ and $f$ is an even function.

A three-layer beam is formed by parallel layers of different materials. For an equally loaded beam of this type, Krajcinovic in [17] proved that the deflection $u$ is governed by the third order differential equation

$$
-u^{\prime \prime \prime}+k^{2} u^{\prime}=a,
$$

where $k$ and $a$ are physical parameters depending on the elasticity of the layers. 
Study of existence of positive solutions for third-order bvps has received a great deal of attention and was the subject of many articles, see, for instance, $[10,11,12,13,14,21,25,27,28,29,30,31]$, for the case of finite intervals and $[1,2,3,4,6,7,8,9,16,19,20,24,26]$ for the case posed on the halfline. Naturally, in such boundary value problems, the nonlinearity may have a singular dependence on time or on the space variable. This was the case in the papers $[3,6,7,8,20,21,27,28,29]$, which motivated this work.

We are concerned in this paper by existence of a positive solution to the boundary value problem (bvp for short),

$$
\left\{\begin{array}{l}
-u^{\prime \prime \prime}(t)+k^{2} u^{\prime}(t)=\phi(t) f\left(t, u(t), u^{\prime}(t)\right), t>0 \\
u(0)=u^{\prime}(0)=u^{\prime}(+\infty)=0
\end{array}\right.
$$

where $k$ is a positive constant, $\phi:(0,+\infty) \rightarrow \mathbb{R}^{+}$is a measurable function, $f: \mathbb{R}^{+} \times(0,+\infty) \times(0,+\infty) \rightarrow \mathbb{R}^{+}$is a continuous function and observe that the form of the differential equation in (5) is more general to those of (1)-(4). Here the constant $k$ which may have a physical signification as in (4), will play an important role in finding a suitable framework for a fixed point formulation of bvp (5).

By positive solution to the bvp (5), we mean a function $u \in C^{2}\left(\mathbb{R}^{+}\right) \cap$ $W^{3,1}(0,+\infty)$ such that $u>0$ in $(0,+\infty)$ and $u(0)=u^{\prime}(0)=\lim _{t \rightarrow+\infty} u^{\prime}(t)=$ 0 , satisfying the differential equation in (5).

In all this paper, we let

$$
\begin{aligned}
& \gamma_{1}(t)=\left(e^{2 k t}-1\right) e^{-4 k t} \\
& \widetilde{\gamma}(t)=k^{*} e^{k t} \gamma_{1}(t)=k^{*}\left(1-e^{-k t}\right)\left(1+e^{-k t}\right) e^{-k t} \\
& \gamma(t)=\int_{0}^{t} \widetilde{\gamma}(s) d s=\frac{k^{*}}{3 k}\left(2-3 e^{-k t}+e^{-3 k t}\right)=\frac{k^{*}}{3 k}\left(1-e^{-k t}\right)^{2}\left(2+e^{-k t}\right)
\end{aligned}
$$

where $k^{*}=\min (1, k) / 2$ and we assume that the functions $\phi$ and $f$ satisfy the following condition:

$$
\left\{\begin{array}{l}
\text { for all } R>0 \text { there exists a function } \Psi_{R}:(0,+\infty) \times(0,+\infty) \rightarrow(0,+\infty) \\
\text { such that } \Psi_{R} \text { nonincreasing following its two variables, } \\
f\left(t, e^{k t} w, e^{k t} z\right) \leq \Psi_{R}(w, z) \text { for all } t, w, z \geq 0 \text { with }|(w, z)| \leq R \\
\lim _{s \rightarrow+\infty} \phi(s) \Psi_{R}\left(r e^{-k s} \gamma(s), r e^{-k s} \widetilde{\gamma}(s)\right)=0 \text { and } \\
\int_{0}^{+\infty} \phi(s) \Psi_{R}\left(r e^{-k s} \gamma(s), r e^{-k s} \widetilde{\gamma}(s)\right) d s<\infty \text { for all } r \in(0, R]
\end{array}\right.
$$


Remark 1 Notice that functions $\mathrm{m}$ in $\mathrm{L}^{1}(0,+\infty)$ do not satisfy $\lim _{\mathrm{t} \rightarrow+\infty}$ $\mathrm{m}(\mathrm{t})=0$. Indeed, the function

$$
m_{0}(t)=\left\{\begin{array}{lll}
2 n^{4} t-n\left(2 n^{4}-1\right) & \text { if } & t \in\left[n-\frac{1}{2 n^{3}}, n\right] \\
-2 n^{4} t+n\left(2 n^{4}+1\right) & \text { if } & t \in\left[n, n+\frac{1}{2 n^{3}}\right] \\
0 & \text { if not } &
\end{array}\right.
$$

is integrable since $\int_{0}^{+\infty} m_{0}(t) d t \leq \sum_{n \geq 1} \frac{1}{n^{2}}<\infty$, and $\lim _{n \rightarrow+\infty} m_{0}(n)=$ $\lim _{\mathrm{n} \rightarrow+\infty} \mathrm{n}=+\infty$.

Hence, the condition $\int_{0}^{+\infty} \phi(\mathrm{s}) \Psi_{\mathrm{R}}\left(\mathrm{r} \mathrm{e}^{-\mathrm{ks}} \gamma(\mathrm{s}), \mathrm{r} \mathrm{e}^{-\mathrm{ks}} \widetilde{\gamma}(\mathrm{s})\right) \mathrm{ds}<\infty$ in Hypothesis (6) does not imply that $\lim _{\mathrm{s} \rightarrow+\infty} \phi(\mathrm{s}) \Psi_{\mathrm{R}}\left(\mathrm{r} \mathrm{e}^{-\mathrm{ks}} \gamma(\mathrm{s}), \mathrm{r} e^{-\mathrm{ks}} \widetilde{\gamma}(\mathrm{s})\right)=0$.

Remark 2 Observe that the case where the nonlinearity $f$ satisfies the polynomial growth condition

$$
f(t, u, v) \leq C\left(1+u^{\sigma}+v^{\mu}\right)
$$

with $\mathrm{c}, \sigma, \mu>0, \lim _{\mathrm{s} \rightarrow+\infty} \phi(\mathrm{s})=0$ and $\int_{0}^{+\infty} \phi(\mathrm{s}) \mathrm{ds}<\infty$, is a particular case where Condition (6) is satisfied.

Remark 3 Notice that if Hypothesis (6) holds then $|\phi|_{1}=\int_{0}^{+\infty} \phi(\mathrm{s}) \mathrm{d} s<\infty$. Indeed, for $\mathrm{R}=1$ we have

$$
\infty>\int_{0}^{+\infty} \phi(\mathrm{s}) \Psi_{1}\left(e^{-\mathrm{ks}} \gamma(\mathrm{s}), \mathrm{e}^{-\mathrm{ks}} \widetilde{\gamma}(\mathrm{s})\right) \mathrm{ds} \geq \Psi_{1}\left(\gamma^{+}, \gamma^{+}\right)|\phi|_{1},
$$

where $\gamma^{+}=\max _{\mathrm{s}>0}\left(e^{-\mathrm{ks}}(\gamma(\mathrm{s})+\widetilde{\gamma}(\mathrm{s}))\right)$.

The statement of the main result needs to introduce the following notations. Let

$$
\begin{aligned}
& f^{0}=\limsup _{|(w, z)| \rightarrow 0}\left(\sup _{t \geq 0} \frac{f\left(t, e^{k t} w, e^{k t} z\right)}{w+z}\right), \\
& f^{\infty}=\limsup _{|(w, z)| \rightarrow+\infty}\left(\sup _{t \geq 0} \frac{f\left(t, e^{k t} w, e^{k t} z\right)}{w+z}\right), \\
& f_{0}(\theta)=\liminf _{|(w, z)| \rightarrow 0}\left(\min _{t \in I_{\theta}} \frac{f\left(t, e^{k t} w, e^{k t} z\right)}{w+z}\right), \\
& f_{\infty}(\theta)=\liminf _{|(w, z)| \rightarrow+\infty}\left(\min _{t \in J_{\theta}} \frac{f\left(t, e^{k t} w, e^{k t} z\right)}{w+z}\right),
\end{aligned}
$$

where $|(w, z)|=|w|+|z|$, for $\theta>0 \mathrm{I}_{\theta}=[0, \theta]$ and for $\theta>1 \mathrm{~J}_{\theta}=[1 / \theta, \theta]$. 
Let also,

$$
\begin{aligned}
\Gamma & =\left(\Gamma_{1}+\Gamma_{2}\right)^{-1}, \\
\Theta_{0}(\theta) & =\left(\Theta_{1,0}(\theta)+\Theta_{2,0}(\theta)\right)^{-1} \text { if } \theta>0, \\
\Theta_{\infty}(\theta) & =\left(\Theta_{1, \infty}(\theta)+\Theta_{2, \infty}(\theta)\right)^{-1} \text { if } \theta>1,
\end{aligned}
$$

where

$$
\begin{aligned}
& \Gamma_{1}=\sup _{t>0}\left(e^{-k t} \int_{0}^{+\infty} G(t, s) \phi(s) d s\right), \\
& \Gamma_{2}=\sup _{t>0}\left(e^{-k t} \int_{0}^{+\infty} \widetilde{G}(t, s) \phi(s) d s\right) \text {, } \\
& \Theta_{1,0}(\theta)=\sup _{t>0}\left(e^{-k t} \int_{0}^{\theta} G(t, s) \phi(s) e^{-k s} \gamma(s) d s\right) \text {, } \\
& \Theta_{2,0}(\theta)=\sup _{t>0}\left(e^{-k t} \int_{0}^{\theta} \widetilde{G}(t, s) \phi(s) e^{-k s} \gamma(s) d s\right), \\
& \Theta_{1, \infty}(\theta)=\sup _{t>0}\left(e^{-k t} \int_{1 / \theta}^{\theta} G(t, s) \phi(s) e^{-k s} \gamma(s) d s\right), \\
& \Theta_{2, \infty}(\theta)=\sup _{t>0}\left(e^{-k t} \int_{1 / \theta}^{\theta} \widetilde{G}(t, s) \phi(s) e^{-k s} \gamma(s) d s\right),
\end{aligned}
$$

and notice that Remark 3 guarantees that the constants $\Gamma_{1}$ and $\Gamma_{2}$ are finite.

Theorem 1 Assume that Hypothesis (6) holds and one of the following conditions

$$
\begin{aligned}
& f^{0}<\Gamma, \quad \Theta_{\infty}(\theta)<f_{\infty}(\theta) \text { for some } \theta>1 \\
& f^{\infty}<\Gamma, \quad \Theta_{0}(\theta)<f_{0}(\theta) \text { for some } \theta>0
\end{aligned}
$$

is satisfied. Then the bvp (5) admits at least one positive solution.

Remark 4 For the particular case where $f(t, u, v)=\left(e^{-k t}(u+v)\right)^{\sigma}$ with $\sigma>$ 0 and $\sigma \neq 1$, we have $\mathrm{f}^{0}=0$ and $\mathrm{f}_{\infty}(\theta)=+\infty$ for all $\theta>0$ if $\sigma>1$, and $f^{\infty}=0$ and $f_{0}(\theta)=+\infty$ for all $\theta>0$ if $\sigma<1$. Hence, Conditions (7) and (8) in Theorem 1 correspond to the superlinear case and the sublinear case of the nonlinearity $\mathrm{f}$, respectively. 


\section{Example}

Consider the case of the bvp (5) where $\phi(t)=e^{-\alpha t}, \alpha>0$ and

$$
f(t, u, v)=A\left(\frac{u+v}{e^{k t}+u+v}\right)^{p}+B\left(\frac{u+v}{e^{k t}}\right)^{q},
$$

with $A, B>0, p \leq 1$ and $q \geq 1$.

Thus, for all $t, w, z>0$ we have

$$
f\left(t, e^{k t} w, e^{k t} z\right)=A\left(\frac{w+z}{1+w+z}\right)^{p}+B(w+z)^{q},
$$

and if $|(w, z)|=w+z<R$, then

$$
f\left(t, e^{k t} w, e^{k t} z\right)=A\left(\frac{w+z}{1+w+z}\right)^{p}+B(w+z)^{q} \leq \Psi_{R}(w, z),
$$

where

$$
\Psi_{R}(w, z)=\left\{\begin{array}{l}
A R^{p}+B R^{q} \text { if } p \geq 0, \\
A(w+z)^{p}(1+R)^{-p}+B R^{q} \text { if } p<0 .
\end{array}\right.
$$

Thus, if $p \geq 0$ then

$$
\begin{gathered}
\lim _{s \rightarrow+\infty} \phi(s) \psi_{R}\left(\operatorname{Re}^{-k s} \gamma(s), \operatorname{Re} e^{-k s} \widetilde{\gamma}(s)\right)=\left(A R^{p}+B R^{q}\right) \lim _{s \rightarrow+\infty} e^{-\alpha s}=0, \\
\int_{0}^{+\infty} \phi(s) \psi_{R}\left(\operatorname{Re}^{-k s} \gamma(s), \operatorname{Re}^{-k s} \widetilde{\gamma}(s)\right) d s=\frac{A R^{p}+B R^{q}}{\alpha}<\infty
\end{gathered}
$$

and if $p<0$ then

$$
\begin{aligned}
\phi(s) \psi_{R}\left(\operatorname{Re}^{-k s} \gamma(s), \operatorname{Re}^{-k s} \widetilde{\gamma}(s)\right)=B R^{q} e^{-\alpha s}+ \\
A(1+R)^{-p}\left(k^{*} R\right)^{p} e^{-(\alpha+p k) s}\left(1-e^{-k s}\right)^{p} \rho(s),
\end{aligned}
$$

where

$$
\rho(s)=\left(\frac{1}{3 k}\left(1-e^{-k s}\right)\left(2+e^{-k s}\right)+e^{-k s}\left(1+e^{-k s}\right)\right)^{p}
$$

satisfies

$$
\left(\max \left(2, \frac{2}{3 k}\right)\right)^{p} \leq \rho(s) \leq\left(\min \left(2, \frac{2}{3 k}\right)\right)^{p} .
$$

Therefore, we have

$$
\lim _{s \rightarrow+\infty} \phi(s) \psi_{R}\left(\operatorname{Re}^{-k s} \gamma(s), \operatorname{Re}^{-k s} \widetilde{\gamma}(s)\right)=0 \text { if and only if } \alpha>-p k
$$


and

$$
\begin{array}{r}
\int_{0}^{+\infty} \phi(s) \psi_{\mathrm{R}}\left(\operatorname{Re}^{-k s} \gamma(s), \operatorname{Re}^{-k s} \widetilde{\gamma}(s)\right) \mathrm{d} s<\infty \text { if and only if } \\
\alpha>-p k \text { and } p>-1 .
\end{array}
$$

Straightforward computations lead to

$$
\begin{aligned}
& f^{\infty}=f_{\infty}(\theta)=f_{\infty}=\left\{\begin{array}{lll}
+\infty & \text { if } & q>1, \\
B & \text { if } & q=1,
\end{array} \quad \text { for all } \theta>1\right. \\
& f^{0}=f_{0}(\theta)=f_{0}=\left\{\begin{array}{lll}
+\infty & \text { if } & p<1, \\
A & \text { if } & p=1<q, \\
A+B & \text { if } & p=q=1,
\end{array} \quad \text { for all } \theta>0\right.
\end{aligned}
$$

We conclude from Theorem 1 and all the above calculations that this case of the bvp (5) admits a positive solution in each of the following situations:

1. $p=1, q=1, B<\Gamma$ and $A+B>\Theta_{0}(\theta)$ for some $\theta>0$,

2. $p=1, q>1$, and $A>\Theta_{0}(\theta)$ for some $\theta>0$,

3. $p \in[0,1), q=1$ and $B<\Gamma$,

4. $p \in(-1,0), q=1, B<\Gamma$ and $\alpha>-p k$.

\section{Abstract background}

Let $(E,\|\|$.$) be a real Banach space. A nonempty closed convex subset C$ of $E$ is said to be a cone in $E$ if $C \cap(-C)=\left\{0_{E}\right\}$ and $t C \subset C$ for all $t \geq 0$.

Let $\Omega$ be a nonempty subset in E. A mapping $A: \Omega \rightarrow E$ is said to be compact if it is continuous and $A(\Omega)$ is relatively compact in $E$.

The main tool of this work is the following Guo-Krasnoselskii's version of expansion and compression of a cone principal in a Banach space.

Theorem 2 Let $\mathrm{P}$ be a cone in $\mathrm{E}$ and let $\Omega_{1}, \Omega_{2}$ be bounded open subsets of $\mathrm{E}$ with $0 \in \Omega_{1}$ and $\bar{\Omega}_{1} \subset \Omega_{2}$. If $\mathrm{T}: \mathrm{P} \cap\left(\bar{\Omega}_{2} \backslash \Omega_{1}\right) \rightarrow \mathrm{P}$ is a compact mapping such that either

1. $\|\mathrm{Tu}\| \leq\|\mathrm{u}\|$ for $\mathrm{u} \in \mathrm{P} \cap \partial \Omega_{1}$ and $\|\mathrm{Tu}\| \geq\|\mathrm{u}\|$ for $\mathrm{u} \in \mathrm{P} \cap \partial \Omega_{2}$, or

2. $\|\mathrm{Tu}\| \geq\|\mathrm{u}\|$ for $\mathrm{u} \in \mathrm{P} \cap \partial \Omega_{1}$ and $\|\mathrm{T} u\| \leq\|u\|$ for $\mathrm{u} \in \mathrm{P} \cap \partial \Omega_{2}$.

Then $\mathrm{T}$ has at least one fixed point in $\mathrm{P} \cap\left(\bar{\Omega}_{2} \backslash \Omega_{1}\right)$. 


\section{Fixed point formulation}

In all this paper, we let

$$
E=\left\{u \in C^{1}\left(\mathbb{R}^{+}, \mathbb{R}\right): \lim _{t \rightarrow+\infty} e^{-k t} u(t)=0, \lim _{t \rightarrow+\infty} e^{-k t} u^{\prime}(t)=0\right\} .
$$

Endowed with the norm $\|u\|=\|u\|_{k}+\left\|u^{\prime}\right\|_{k}$ where $\|u\|_{k}=\sup _{t \geq 0}\left(e^{-k t}|u(t)|\right)$, E becomes a Banach space.

The following lemma is an adapted version for the case of the space $E$ of Corduneanu's compactness criterion ([5], p. 62). It will be used in this work to prove that some operator is completely continuous.

Lemma 1 A nonempty subset $\mathrm{M}$ of $\mathrm{E}$ is relatively compact if the following conditions hold:

(a) $\mathrm{M}$ is bounded in $\mathrm{E}$,

(b) the sets $\left\{u: u(t)=e^{-k t} \chi(t), x \in M\right\}$ and $\left\{u: u(t)=e^{-k t} x^{\prime}(t), x \in M\right\}$ are locally equicontinuous on $[0,+\infty)$, and

(c) the sets $\left\{u: u(t)=e^{-k t} x(t), x \in M\right\}$ and $\left\{u: u(t)=e^{-k t} x^{\prime}(t), x \in M\right\}$ are equiconvergent at $+\infty$.

In all this work, $\mathrm{P}$ denotes the cone in $\mathrm{E}$ defined by

$$
P=\left\{u \in E: u^{\prime}(t) \geq \widetilde{\gamma}(t)\|u\| \text { and } u(t) \geq \gamma(t)\|u\| \text { for all } t>0\right\} .
$$

Let $\mathrm{G}, \widetilde{\mathrm{G}}: \mathbb{R}^{+} \times \mathbb{R}^{+} \rightarrow \mathbb{R}^{+}$be the functions defined by

$$
\begin{aligned}
& G(t, s)=\frac{1}{k^{2}} \begin{cases}e^{-k s}(\cosh (k t)-1) & \text { if } t \leq s, \\
-e^{-k t} \sinh (k s)+\left(1-e^{-k s}\right) & \text { if } s \leq t,\end{cases} \\
& \widetilde{G}(t, s)=\frac{\partial G}{\partial t}(t, s)=\frac{1}{k}\left\{\begin{array}{lll}
e^{-k s} \sinh (k t) & \text { if } t \leq s \\
e^{-k t} \sinh (k s) & \text { if } \quad s \leq t .
\end{array}\right.
\end{aligned}
$$

Lemma 2 The functions $\mathrm{G}$ and $\widetilde{\mathrm{G}}$ satisfy:

(a) For all $\mathrm{t}, \mathrm{s} \in \mathbb{R}^{+}$we have $\mathrm{G}(\mathrm{t}, \mathrm{s}) \geq 0$ and $\widetilde{\mathrm{G}}(\mathrm{t}, \mathrm{s}) \geq 0$.

(b) The functions $\mathrm{G}$ and $\widetilde{\mathrm{G}}$ are continuous and for all $\mathrm{s} \geq 0$, we have

$$
\mathrm{G}(0, s)=\widetilde{\mathrm{G}}(0, s)=0 .
$$


(c) For all $\mathrm{t}, \mathrm{s} \geq 0$, we have

$$
\mathrm{G}(\mathrm{t}, \mathrm{s}) \leq \frac{1}{\mathrm{k}^{2}}\left(1-e^{-\mathrm{ks}}\right) \leq \frac{1}{\mathrm{k}^{2}}, \quad \widetilde{\mathrm{G}}(\mathrm{t}, \mathrm{s}) \leq \widetilde{\mathrm{G}}(\mathrm{s}, \mathrm{s}) \leq \frac{1}{2 \mathrm{k}} .
$$

(d) For all s, t, $\tau \geq 0$, we have

$$
\widetilde{\mathrm{G}}(\mathrm{t}, \mathrm{s}) e^{-k t} \geq \gamma_{1}(\mathrm{t}) \widetilde{\mathrm{G}}(\tau, s) e^{-k \tau} .
$$

(e) For all $\mathrm{t}_{2}, \mathrm{t}_{1} \geq 0$, we have

$$
\begin{gathered}
\left|e^{-k t_{2}} G\left(t_{2}, s\right)-e^{-k t_{1}} G\left(t_{1}, s\right)\right| \leq \frac{3}{2 k}\left|t_{2}-t_{1}\right| \\
\left|e^{-k t_{2}} \widetilde{G}\left(t_{2}, s\right)-e^{-k t_{1}} \widetilde{G}\left(t_{1}, s\right)\right| \leq\left|t_{2}-t_{1}\right|
\end{gathered}
$$

Proof. Assertions (a), (b) and (c) are easy to prove, Assertion (d) is proved in [8]. Assertion (e) is obtained by the mean value theorem.

Lemma 3 Assume that Hypothesis (6) holds, then there exists a continuous operator $\mathrm{T}: \mathrm{P} \backslash\{0\} \rightarrow \mathrm{P}$ such that for all $\mathrm{r}, \mathrm{R}$ with $0<\mathrm{r}<\mathrm{R}, \mathrm{T}(\mathrm{P} \cap(\overline{\mathrm{B}}(0, \mathrm{R}) \backslash$ $\mathrm{B}(0, \mathrm{r}))$ ) is relatively compact and fixed points of $\mathrm{T}$ are positive solutions to the bvp (5).

Proof. The proof is divided into four steps.

Step 1. In this step we prove the existence of the operator T. To this aim let $u \in P \backslash\{0\}$. By means of Hypothesis (6) with $R=\|u\|$, for all $t>0$ we have

$$
\begin{aligned}
\int_{0}^{+\infty} \mathrm{G}(\mathrm{t}, \mathrm{s}) \phi(s) f(s, & \left.\mathrm{u}(\mathrm{s}), \mathrm{u}^{\prime}(\mathrm{s})\right) \mathrm{d} s \\
& \leq \frac{1}{\mathrm{k}^{2}} \int_{0}^{+\infty} \phi(s) f\left(s, u(s), u^{\prime}(s)\right) d s \\
& =\frac{1}{k^{2}} \int_{0}^{+\infty} \phi(s) f\left(s, e^{k s}\left(e^{-k s} u(s)\right), e^{k s}\left(e^{-k s} u^{\prime}(s)\right)\right) d s \\
& \leq \frac{1}{k^{2}} \int_{0}^{+\infty} \phi(s) \Psi_{R}\left(\operatorname{Re}^{-k s} \gamma(s), \operatorname{Re}^{-k s} \widetilde{\gamma}(s)\right) d s<\infty
\end{aligned}
$$

and

$$
\int_{0}^{+\infty} \widetilde{G}(t, s) \phi(s) f\left(s, u(s), u^{\prime}(s)\right) d s \leq \frac{1}{2 k} \int_{0}^{+\infty} \phi(s) f\left(s, u(s), u^{\prime}(s)\right) d s
$$




$$
\leq \frac{1}{2 k} \int_{0}^{+\infty} \phi(s) \Psi_{R}\left(\operatorname{Re}^{-k s} \gamma(s), \operatorname{Re}^{-k s} \widetilde{\gamma}(s)\right) \mathrm{d} s<\infty
$$

Thus, let $v$ and $w$ be the functions defined by

$$
\begin{aligned}
& v(t)=\int_{0}^{+\infty} G(t, s) \phi(s) f\left(s, u(s), u^{\prime}(s)\right) d s \\
& w(t)=\int_{0}^{+\infty} \widetilde{G}(t, s) \phi(s) f\left(s, u(s), u^{\prime}(s)\right) d s .
\end{aligned}
$$

Since for all $t>0$,

$$
\begin{aligned}
v(t)= & -\frac{e^{-k t}}{k^{2}} \int_{0}^{t} \sinh (k s) \phi(s) f\left(s, u(s), u^{\prime}(s)\right) d s \\
& +\frac{1}{k^{2}} \int_{0}^{t}\left(1-e^{-k s}\right) \phi(s) f\left(s, u(s), u^{\prime}(s)\right) d s \\
& +\frac{\cosh (k t)-1}{k^{2}} \int_{0}^{t} e^{-k s} \phi(s) f\left(s, u(s), u^{\prime}(s)\right) d s,
\end{aligned}
$$

we see that $v$ is differentiable on $\mathbb{R}^{+}$and for all $t \geq 0$,

$$
\begin{aligned}
v^{\prime}(t)= & \frac{e^{-k t}}{k} \int_{0}^{t} \sinh (k s) \phi(s) f\left(s, u(s), u^{\prime}(s)\right) d s \\
& +\frac{\sinh (k t)}{k} \int_{t}^{+\infty} e^{-k s} \phi(s) f\left(s, u(s), u^{\prime}(s)\right) d s \\
= & \int_{0}^{+\infty} \widetilde{G}(t, s) \phi(s) f\left(s, u(s), u^{\prime}(s)\right) d s=w(t)
\end{aligned}
$$

with $w$ continuous on $\mathbb{R}^{+}$.

At this stage we have proved that $v$ belongs to $C^{1}\left(\mathbb{R}^{+}, \mathbb{R}\right)$ and we need to prove that $v \in \mathrm{E}$. Thus, we have to show that $\lim _{\mathrm{t} \rightarrow+\infty} \mathrm{e}^{-\mathrm{kt}} v(\mathrm{t})=\lim _{\mathrm{t} \rightarrow+\infty}$ $e^{-k t} v^{\prime}(t)=0$. Clearly for all $t>0, v(t), v^{\prime}(t)>0$ and we have

$$
\begin{aligned}
e^{-k t} v(t) & =e^{-k t} \int_{0}^{+\infty} G(t, s) \phi(s) f\left(s, u(s), u^{\prime}(s)\right) d s \\
& \leq \frac{e^{-k t}}{k^{2}} \int_{0}^{+\infty} \phi(s) \Psi_{R}\left(\operatorname{Re}^{-k s} \gamma(s), \operatorname{Re}^{-k s} \widetilde{\gamma}(s)\right) d s
\end{aligned}
$$

and

$$
e^{-k t} v^{\prime}(t)=e^{-k t} \int_{0}^{+\infty} \widetilde{G}(t, s) \phi(s) f\left(s, u(s), u^{\prime}(s)\right) d s
$$




$$
\leq \frac{e^{-k t}}{2 k} \int_{0}^{+\infty} \phi(s) \Psi_{R}\left(\operatorname{Re}^{-k s} \gamma(s), \operatorname{Re}^{-k s} \gamma(s)\right) \mathrm{ds}
$$

The above two estimates prove that $\lim _{\mathrm{t} \rightarrow+\infty} e^{-k \mathrm{t}} v(\mathrm{t})=\lim _{\mathrm{t} \rightarrow+\infty} e^{-k \mathrm{t}} v^{\prime}(\mathrm{t})=$ 0 .

Now for all $t, \tau>0$, we have from Assertion (c) in Lemma 2

$$
\begin{aligned}
v^{\prime}(t) & =e^{k t} \int_{0}^{+\infty} e^{-k t} \widetilde{G}(t, s) f\left(s, u(s), u^{\prime}(s)\right) d s \\
& \geq e^{k t} \gamma_{1}(t) \int_{0}^{+\infty} e^{-k \tau} \widetilde{G}(\tau, s) f\left(s, u(s), u^{\prime}(s)\right) d s \\
& =e^{k t} \gamma_{1}(t) e^{-k \tau} v^{\prime}(\tau) .
\end{aligned}
$$

Passing to the supremum on $\tau$, we obtain

$$
v^{\prime}(t) \geq e^{k t} \gamma_{1}(t)\left\|v^{\prime}\right\|_{k} \text { for all } t>0
$$

Since for all $t>0$

$$
v(t)=\int_{0}^{t} e^{k \xi}\left(e^{-k \xi} v^{\prime}(\xi)\right) d \xi \leq \int_{0}^{t} e^{k \xi} d \xi\left\|v^{\prime}\right\|_{k} \leq \frac{e^{k t}}{k}\left\|v^{\prime}\right\|_{k},
$$

we have

$$
\left\|v^{\prime}\right\|_{k} \geq k\|v\|_{k} \text {. }
$$

Therefore, (12) Combined with (13) leads to

$$
v^{\prime}(t) \geq k e^{k t} \gamma_{1}(t)\left\|v^{\prime}\right\|_{k} \text { for all } t>0,
$$

then to

$$
v^{\prime}(\mathrm{t}) \geq \widetilde{\gamma}(\mathrm{t})\|v\| \text { for all } \mathrm{t}>0 .
$$

Integrating (14), yields $v(t) \geq \gamma(t)\|v\|$ for all $t>0$.

Thus, we have proved that $v \in \mathrm{P}$ and the operator $\mathrm{T}: \mathrm{P} \backslash\{0\} \rightarrow \mathrm{P}$ where for $u \in P \backslash\{0\}$

$$
T u(t)=\int_{0}^{+\infty} G(t, s) \phi(s) f\left(s, u(s), u^{\prime}(s)\right) d s,
$$

is well defined.

Step 2. In this step we prove that the operator $T$ is continuous. Let $\left(u_{n}\right)$ be a sequence in $P \backslash\{0\}$ such that $\lim _{n \rightarrow \infty} u_{n}=u_{\infty}$ in $E$ with $u_{\infty}$ in $P \backslash\{0\}$ 
and let $R>r>0$ be such that $\left(u_{n}\right) \subset B(0, R) \backslash B(0, r)$. If $\Psi_{R}$ is the function given by Hypothesis (6), then for all $n \geq 1$ we have

$$
\begin{aligned}
\left\|T u_{n}-T u_{\infty}\right\|_{k} & =\sup _{t \geq 0}\left|T u_{n}(t)-T u_{\infty}(t)\right| \\
& \leq \frac{1}{k^{2}} \int_{0}^{+\infty} \phi(s)\left|f\left(s, u_{n}(s), u_{n}^{\prime}(s)\right)-f\left(s, u_{\infty}(s), u_{\infty}^{\prime}(s)\right)\right| d s
\end{aligned}
$$

and

$$
\begin{aligned}
\left\|\left(T u_{n}\right)^{\prime}-\left(T u_{\infty}\right)^{\prime}\right\|_{k} & =\sup _{t \geq 0}\left|\left(T u_{n}\right)^{\prime}(t)-\left(T u_{\infty}\right)^{\prime}(t)\right| \\
& \leq \frac{1}{2 k} \int_{0}^{+\infty} \phi(s)\left|f\left(s, u_{n}(s), u_{n}^{\prime}(s)\right)-f\left(s, u_{\infty}(s), u_{\infty}^{\prime}(s)\right)\right| d s .
\end{aligned}
$$

Because of

$$
\left|f\left(s, u_{n}(s), u_{n}^{\prime}(s)\right)-f\left(s, u_{\infty}(s), u_{\infty}^{\prime}(s)\right)\right| \rightarrow 0, \text { as } n \rightarrow+\infty
$$

for all $s>0$ and

$$
\begin{aligned}
& \phi(s)\left|f\left(s, u_{n}(s), u_{n}^{\prime}(s)\right)-f\left(s, u_{\infty}(s), u_{\infty}^{\prime}(s)\right)\right| \\
& \leq 2 \phi(s) \Psi_{R}\left(r e^{-k s} \gamma(s), r e^{-k s} \gamma(s)\right)
\end{aligned}
$$

with $\int_{0}^{+\infty} \phi(s) \Psi_{R}\left(r e^{-k s} \gamma(s), r e^{-k s} \gamma(s)\right) d s<\infty$, the Lebesgue dominated convergence theorem guarantees that $\lim _{n \rightarrow \infty}\left\|T u_{n}-T u_{\infty}\right\|=0$. Hence, we have proved that $\mathrm{T}$ is continuous.

Step 3. In this step, we prove that for $R>r>0, T(P \cap(\bar{B}(0, R) \backslash B(0, r)))$ is relatively compact. Set $\Omega=\mathrm{P} \cap(\overline{\mathrm{B}}(0, R) \backslash \mathrm{B}(0, r))$ and let $\Phi_{r, R}$ be defined by

$$
\Phi_{r, R}(s)=\Psi_{R}\left(r e^{-k s} \gamma(s), r e^{-k s} \gamma(s)\right)
$$

where $\Psi_{R}$ is the function given by Hypothesis (6). For all $u \in \Omega$, we have

$$
\|\mathrm{Tu}\| \leq\left(\frac{1}{\mathrm{k}^{2}}+\frac{1}{2 \mathrm{k}}\right) \int_{0}^{+\infty} \phi(\mathrm{s}) \Phi_{\mathrm{r}, \mathrm{R}}(\mathrm{s}) \mathrm{d} \mathrm{s}<\infty,
$$

proving that $T \Omega$ is bounded in $E$.

Now, let $t_{1}, t_{2} \in[\eta, \xi]$, for all $u \in \Omega$, we have from (10) and (11) the estimates $\left|e^{-k t_{1}} T u\left(t_{1}\right)-e^{-k t_{2}} T u\left(t_{2}\right)\right| \leq \int_{0}^{+\infty}\left|e^{-k t_{1}} G\left(t_{1}, s\right)-e^{-k t_{2}} G\left(t_{2}, s\right)\right| \phi(s) \Phi_{r, R}(s) d s$ 


$$
\leq \frac{3}{2 k}\left|t_{2}-t_{1}\right| \int_{0}^{+\infty} \phi(s) \Phi_{r, R}(s) d s
$$

and

$$
\begin{aligned}
\left|e^{-k t_{1}}(T u)^{\prime}\left(t_{1}\right)-e^{-k t_{2}}(T u)^{\prime}\left(t_{2}\right)\right| & \leq \int_{0}^{+\infty}\left|e^{-k t_{1}} \widetilde{G}\left(t_{1}, s\right)-e^{-k t_{2}} \widetilde{G}\left(t_{2}, s\right)\right| \phi(s) \Phi_{r, R}(s) d s \\
& \leq\left|t_{2}-t_{1}\right| \int_{0}^{+\infty} \phi(s) \Phi_{r, R}(s) d s .
\end{aligned}
$$

Proving the equicontinuity of $T \Omega$ on bounded intervals.

For all $u \in \Omega$ and $\mathrm{t}>0$, we have

$$
\left|e^{-k t} \operatorname{Tu}(t)\right| \leq \frac{e^{-k t}}{k^{2}} \int_{0}^{+\infty} \phi(s) \Phi_{r, R}(s) d s
$$

and

$$
\left|e^{-k t}(T u)^{\prime}(t)\right| \leq \frac{e^{-k t}}{k} \int_{0}^{+\infty} \phi(s) \Phi_{r, R}(s) d s .
$$

Thus, the equiconvergence of $T \Omega$ follows from the fact that $\lim _{t \rightarrow \infty} e^{-k t}=0$. In view of Lemma $1, T \Omega$ is relatively compact in $E$.

Step 4. In this step we prove that fixed points of $T$ are positive solutions to the bvp (5). Let $u \in P \backslash\{0\}$ be a fixed point of $T$, then for all $t>0$ we have

$$
\begin{aligned}
u(t) & =\int_{0}^{+\infty} G(t, s) \phi(s) f\left(s, u(s), u^{\prime}(s)\right) d s \text { and } \\
u^{\prime}(t) & =\int_{0}^{+\infty} \widetilde{G}(t, s) \phi(s) f\left(s, u(s), u^{\prime}(s)\right) d s .
\end{aligned}
$$

These with (9) lead to $\mathfrak{u}(0)=\mathfrak{u}^{\prime}(0)=0$.

Differentiating twice in

$$
\begin{aligned}
u^{\prime}(t)= & \int_{0}^{+\infty} \widetilde{G}(t, s) \phi(s) f\left(s, u(s), u^{\prime}(s)\right) d s \\
= & \frac{e^{-k t}}{k} \int_{0}^{t} \sinh (k s) \phi(s) f\left(s, u(s), u^{\prime}(s)\right) d s \\
& +\frac{\sinh (k t)}{k} \int_{t}^{+\infty} e^{-k s} \phi(s) f\left(s, u(s), u^{\prime}(s)\right) d s
\end{aligned}
$$

we see that $-u^{\prime \prime \prime}(t)+k u^{\prime}(t)=\phi(t) f\left(t, u(t), u^{\prime}(t)\right)$ for all $t>0$. 
It remains to prove that $\lim _{t \rightarrow+\infty} u^{\prime}(t)=0$. We have

$$
\begin{aligned}
u^{\prime}(t)= & \frac{1}{k e^{k t}} \int_{0}^{t} \sinh (k s) \phi(s) f\left(s, u(s), u^{\prime}(s)\right) d s \\
& +\frac{\sinh (k t)}{k} \int_{t}^{+\infty} e^{-k s} \phi(s) f\left(s, u(s), u^{\prime}(s)\right) d s .
\end{aligned}
$$

By means of Hypothesis (6) with $R=\|u\|$ and the L'Hopital's rule, we obtain

$$
\begin{aligned}
& \lim _{t \rightarrow+\infty} \frac{1}{k e^{k t}} \int_{0}^{t} \sinh (k s) \phi(s) f\left(s, u(s), u^{\prime}(s)\right) d s \\
& \quad \leq \lim _{t \rightarrow+\infty} \frac{1}{k e^{k t}} \int_{0}^{t} \sinh (k s) \phi(s) \Psi_{R}\left(\operatorname{Re}^{-k s} \gamma(s), \operatorname{Re}^{-k s} \gamma(s)\right) d s \\
& \quad=\lim _{t \rightarrow+\infty} \frac{\sinh (k t)}{k e^{k t}} \phi(t) \Psi_{R}\left(\operatorname{Re}^{-k t} \gamma(t), \operatorname{Re}^{-k t} \gamma(t)\right) d s=0 .
\end{aligned}
$$

Also, we have

$$
\begin{aligned}
& \frac{\sinh (k t)}{k} \int_{t}^{+\infty} e^{-k s} \phi(s) f\left(s, u(s), u^{\prime}(s)\right) d s \\
& \leq \frac{\sinh (k t) e^{-k t}}{k} \int_{t}^{+\infty} \phi(s) f\left(s, u(s), u^{\prime}(s)\right) d s \rightarrow 0 \text { as } t \rightarrow+\infty .
\end{aligned}
$$

The above calculations show that $\lim _{t \rightarrow+\infty} \mathfrak{u}^{\prime}(t)=0$, completing the proof of the lemma.

\section{Proof of Theorem 1}

\section{Step 1. Existence in the case where (7) holds}

Let $\epsilon>0$ be such that $\left(f^{0}+\epsilon\right)<\Gamma$. For such a $\epsilon$, there exists $R_{1}>0$ such that $f\left(t, e^{k t} w, e^{k t} z\right) \leq\left(f^{0}+\epsilon\right)(w+z)$ for all $w, z$ with $|(w, z)| \leq R_{1}$ and let $\Omega_{1}=\left\{\mathrm{u} \in \mathrm{E},\|\mathrm{u}\|<\mathrm{R}_{1}\right\}$.

Therefore, for all $u \in P \cap \partial \Omega_{1}$ and all $t>0$, we have

$$
\begin{aligned}
e^{-k t} T u(t) & =e^{-k t} \int_{0}^{+\infty} G(t, s) \phi(s) f\left(s, e^{k s}\left(e^{-k s} u(s)\right), e^{k s}\left(e^{-k s} u^{\prime}(s)\right)\right) d s \\
& \leq\left(f^{0}+\epsilon\right) e^{-k t} \int_{0}^{+\infty} G(t, s) \phi(s) e^{-k s}\left(u(s)+u^{\prime}(s)\right) d s \\
& \leq\|u\|\left(f^{0}+\epsilon\right) e^{-k t} \int_{0}^{+\infty} G(t, s) \phi(s) d s \leq \Gamma_{1}\left(f^{0}+\epsilon\right)\|u\|,
\end{aligned}
$$


leading to

$$
\|T u\|_{k}=\sup _{t>0}\left(e^{-k t} T u(t)\right) \leq\left(f^{0}+\epsilon\right) \Gamma_{1}\|u\|
$$

Similarly, we have

$$
\begin{aligned}
e^{-k t}(T u)^{\prime}(t) & =e^{-k t} \int_{0}^{+\infty} \widetilde{G}(t, s) \phi(s) f\left(s, e^{k s}\left(e^{-k s} u(s)\right), e^{k s}\left(e^{-k s} u^{\prime}(s)\right)\right) d s \\
& \leq\left(f^{0}+\epsilon\right) e^{-k t} \int_{0}^{+\infty} \widetilde{G}(t, s) \phi(s) e^{-k s}\left(u(s)+u^{\prime}(s)\right) d s \\
& \leq\|u\|\left(f^{0}+\epsilon\right) e^{-k t} \int_{0}^{+\infty} \widetilde{G}(t, s) \phi(s) d s \\
& \leq\left(f^{0}+\epsilon\right) \Gamma_{2}\|u\|,
\end{aligned}
$$

leading to

$$
\left\|(T u)^{\prime}\right\|_{k}=\sup _{t>0}\left(e^{-k t}(T u)^{\prime}(t)\right) \leq\left(f^{0}+\epsilon\right) \Gamma_{2}\|u\|
$$

Summing (15) with (16), we obtain

$$
\|T u\| \leq\|u\|\left(f^{0}+\epsilon\right) \Gamma^{-1} \leq\|u\| .
$$

Now, suppose that $f_{\infty}(\theta)>\Theta_{\infty}(\theta)$ for some $\theta>1$ and let $\varepsilon>0$ be such that

$\left(f_{\infty}(\theta)-\varepsilon\right)>\Theta_{\infty}(\theta)$. There exists $\widetilde{R}_{2}>R_{1}$ such that $f\left(t, e^{k t} w, e^{k t} z\right)>$ $\left(f_{\infty}(\theta)-\varepsilon\right)(w+z)$ for all $t \in J_{\theta}$ and all $w, z$ with $|(w, z)| \geq \widetilde{R}_{2}$. Let $\gamma_{\theta}=$ $\min \left\{\gamma(s) e^{-k s}: s \in J_{\theta}\right\}, R_{2}=\widetilde{R}_{2} / \gamma_{\theta}$ and $\Omega_{2}=\left\{u \in E:\|u\|<R_{2}\right\}$. For all $u \in \mathrm{P} \cap \partial \Omega_{2}$, and all $\mathrm{t}>0$ we have

$$
\begin{aligned}
\|T u\|_{k} & \geq e^{-k t} T u(t) \geq e^{-k t} \int_{1 / \theta}^{\theta} G(t, s) \phi(s) f\left(s, e^{k s}\left(e^{-k s} u(s)\right), e^{k s}\left(e^{-k s} u^{\prime}(s)\right)\right) d s \\
& \geq\left(f_{\infty}(\theta)-\varepsilon\right) e^{-k t} \int_{1 / \theta}^{\theta} G(t, s) \phi(s)\left(e^{-k s} u(s)+e^{-k s} u^{\prime}(s)\right) d s \\
& \geq\left(f_{\infty}(\theta)-\varepsilon\right) e^{-k t} \int_{1 / \theta}^{\theta} G(t, s) \phi(s) e^{-k s} u(s) d s \\
& \geq\|u\|\left(f_{\infty}(\theta)-\varepsilon\right) e^{-k t} \int_{1 / \theta}^{\theta} G(t, s) \phi(s) e^{-k s} \gamma(s) d s
\end{aligned}
$$


and

$$
\begin{aligned}
\left\|(T u)^{\prime}\right\|_{k} & \geq e^{-k t} \int_{1 / \theta}^{\theta} \widetilde{G}(t, s) \phi(s) f\left(s, e^{k s}\left(e^{-k s} u(s)\right), e^{k s}\left(e^{-k s} u^{\prime}(s)\right)\right) d s \\
& \geq\left(f_{\infty}(\theta)-\varepsilon\right) e^{-k t} \int_{1 / \theta}^{\theta} \widetilde{G}(t, s) \phi(s)\left(e^{-k s} u(s)+e^{-k s} u^{\prime}(s)\right) d s \\
& \geq\left(f_{\infty}(\theta)-\varepsilon\right) e^{-k t} \int_{1 / \theta}^{\theta} \widetilde{G}(t, s) \phi(s) e^{-k s} u(s) d s \\
& \geq\|u\|\left(f_{\infty}(\theta)-\varepsilon\right) e^{-k t} \int_{1 / \theta}^{\theta} \widetilde{G}(t, s) \phi(s) e^{-k s} \gamma(s) d s .
\end{aligned}
$$

The above estimates lead to

$$
\begin{aligned}
& \|T u\|_{k} \geq\left(f_{\infty}(\theta)-\varepsilon\right) \Theta_{1, \infty}(\theta)\|u\|, \\
& \left\|(T u)^{\prime}\right\|_{k} \geq\left(f_{\infty}(\theta)-\varepsilon\right) \Theta_{2, \infty}(\theta)\|u\|
\end{aligned}
$$

then to

$$
\|T u\| \geq\left(f_{\infty}(\theta)-\varepsilon\right)\left(\Theta_{\infty}(\theta)\right)^{-1}\|u\| \geq\|u\| .
$$

We deduce from Assertion 1 of Theorem 2, that $T$ admits a fixed point $u \in P$ with

$\mathrm{R}_{1} \leq\|\mathrm{u}\|_{1} \leq \mathrm{R}_{2}$ which is, by Lemma 3, a positive solution to Problem (5).

\section{Step 2. Existence in the case where (8) holds}

Suppose that $f_{0}(\theta)>\Theta_{0}(\theta)$ for some $\theta>0$ and let $\varepsilon>0$ be such that $\left(f_{0}(\theta)-\varepsilon\right)>\Theta_{0}(\theta)$. There exists $R_{1}$ such that $f\left(t, e^{k t} w, e^{k t} z\right)>\left(f_{0}(\theta)-\right.$ $\varepsilon)(w+z)$ for all $w, z$ with $|(w, z)| \leq \mathrm{R}_{1}$. Let $\Omega_{1}=\left\{\mathrm{u} \in \mathrm{E}:\|\mathrm{u}\|<\mathrm{R}_{1}\right\}$, for all $u \in P \cap \partial \Omega_{1}$ and all $t>0$, we have

$$
\begin{aligned}
\|T u\|_{k} & \geq e^{-k t} T u(t) \geq e^{-k t} \int_{0}^{\theta} G(t, s) \phi(s) f\left(s, e^{k s}\left(e^{-k s} u(s)\right), e^{k s}\left(e^{-k s} u^{\prime}(s)\right)\right) d s \\
& \geq\left(f_{0}(\theta)-\varepsilon\right) e^{-k t} \int_{0}^{\theta} G(t, s) \phi(s)\left(e^{-k s} u(s)+e^{-k s} u^{\prime}(s)\right) d s \\
& \geq\left(f_{0}(\theta)-\varepsilon\right) e^{-k t} \int_{0}^{\theta} G(t, s) \phi(s) e^{-k s} u(s) d s \\
& \geq\|u\|\left(f_{0}(\theta)-\varepsilon\right) e^{-k t} \int_{0}^{\theta} G(t, s) \phi(s) e^{-k s} \gamma(s) d s
\end{aligned}
$$


and

$$
\begin{aligned}
\left\|(T u)^{\prime}\right\|_{k} & \geq e^{-k t} T u(t) \geq e^{-k t} \int_{0}^{\theta} \widetilde{G}(t, s) \phi(s) f\left(s, e^{k s}\left(e^{-k s} u(s)\right), e^{k s}\left(e^{-k s} u^{\prime}(s)\right)\right) d s \\
& \geq\left(f_{0}(\theta)-\varepsilon\right) e^{-k t} \int_{0}^{\theta} \widetilde{G}(t, s) \phi(s)\left(e^{-k s} u(s)+e^{-k s} u^{\prime}(s)\right) d s \\
& \geq\left(f_{0}(\theta)-\varepsilon\right) e^{-k t} \int_{0}^{\theta} \widetilde{G}(t, s) \phi(s) e^{-k s} u(s) d s \\
& \geq\|u\|\left(f_{0}(\theta)-\varepsilon\right) e^{-k t} \int_{0}^{\theta} \widetilde{G}(t, s) \phi(s) e^{-k s} \gamma(s) d s .
\end{aligned}
$$

The above estimates lead to

$$
\begin{aligned}
& \|T u\|_{k} \geq\left(f_{0}(\theta)-\varepsilon\right) \Theta_{1,0}(\theta)\|u\|, \\
& \left\|(T u)^{\prime}\right\|_{k} \geq\left(f_{0}(\theta)-\varepsilon\right) \Theta_{2,0}(\theta)\|u\|
\end{aligned}
$$

then to

$$
\|\mathrm{Tu}\| \geq\left(\mathrm{f}_{0}(\theta)-\varepsilon\right)\left(\Theta_{0}(\theta)\right)^{-1}\|\mathrm{u}\| \geq\|\mathrm{u}\| .
$$

Let $\epsilon>0$ be such that $\left(f^{\infty}+\epsilon\right)<\Gamma$, there exists $R_{\epsilon}>0$ such that

$$
f\left(t, e^{k t} w, e^{k t} z\right) \leq\left(f^{\infty}+\epsilon\right)(w+z)+\Psi_{R_{\epsilon}}(w, z) \text {, for all } t, w, z>0,
$$

where $\Psi_{R_{\epsilon}}$ is the functions given by Hypothesis (6) for $R=R_{\epsilon}$.

Let

$$
\begin{aligned}
& \Phi_{\epsilon}(t)=\Psi_{R_{\epsilon}}\left(R_{\epsilon} e^{-k s} \gamma(s), R_{\epsilon} e^{-k s} \widetilde{\gamma}(s)\right) \\
& \widetilde{R}_{2}=\frac{2 \Psi_{\epsilon} \Gamma}{\Gamma-(f \infty+\epsilon)} \\
& \text { with } \overline{\Phi_{\epsilon}}=\sup _{t \geq 0}\left(e^{-k t} \int_{0}^{+\infty} G(t, s) \Phi_{\epsilon}(s) d s\right)
\end{aligned}
$$

and notice that $\Gamma^{-1}\left(f^{\infty}+\epsilon\right) R+2 \overline{\Phi_{\epsilon}} \leq R$ for all $R \geq \widetilde{R}_{2}$.

Let $R_{2}>\max \left(R_{1}, \widetilde{R}_{2}, R_{\epsilon}\right)$ and $\Omega_{2}=\left\{u \in E,\|u\|<R_{2}\right\}$. For all $u \in P \cap \partial \Omega_{2}$ and all $t>0$, we have

$$
\begin{aligned}
e^{-k t} T u(t) & =\int_{0}^{+\infty} G(t, s) \phi(s) f\left(s, e^{k s}\left(e^{-k s} u(s)\right), e^{k s}\left(e^{-k s} u^{\prime}(s)\right)\right) d s \\
& \leq e^{-k t} \int_{0}^{+\infty} G(t, s) \phi(s)\left(\left(f^{\infty}+\epsilon\right)\left(e^{-k s} u(s)+e^{-k s} u^{\prime}(s)\right)\right. \\
& \left.+\Psi_{\epsilon}\left(e^{-k s} u(s), e^{-k s} u^{\prime}(s)\right)\right) d s
\end{aligned}
$$




$$
\begin{aligned}
& \leq\left(f^{\infty}+\epsilon\right)\|u\| e^{-k t} \int_{0}^{+\infty} G(t, s) \phi(s) d s+\overline{\Psi_{\epsilon}} \\
& \leq\left(f^{\infty}+\epsilon\right)\|u\| \Gamma_{1}+\overline{\Psi_{\epsilon}}
\end{aligned}
$$

leading to

$$
\|T u\|_{k} \leq\left(f^{\infty}+\epsilon\right)\|u\| \Gamma_{1}+\overline{\Psi_{\epsilon}} .
$$

Similarly, we have

$$
\begin{aligned}
& e^{-k \mathrm{t}}(\mathrm{Tu})^{\prime}(\mathrm{t})=\int_{0}^{+\infty} \widetilde{\mathrm{G}}(\mathrm{t}, \mathrm{s}) \phi(\mathrm{s}) \mathrm{f}\left(\mathrm{s}, e^{\mathrm{ks}}\left(e^{-k s} \mathrm{u}(\mathrm{s})\right), e^{\mathrm{ks}}\left(e^{-k s} u^{\prime}(s)\right)\right) \mathrm{ds} \\
& \leq e^{-k t} \int_{0}^{+\infty} \widetilde{G}(t, s) \phi(s)\left(\left(f^{\infty}+\epsilon\right)\left(e^{-k s} u(s)+e^{-k s} u^{\prime}(s)\right)\right. \\
& \left.+\Psi_{\epsilon}\left(e^{-k s} u(s), e^{-k s} u^{\prime}(s)\right)\right) d s \\
& \leq\left(f^{\infty}+\epsilon\right)\|\mathrm{u}\| e^{-k t} \int_{0}^{+\infty} \widetilde{\mathrm{G}}(\mathrm{t}, \mathrm{s}) \phi(\mathrm{s}) \mathrm{d} s+\overline{\Psi_{\epsilon}} \\
& \leq\left(f^{\infty}+\epsilon\right)\|u\| \Gamma_{2}+\overline{\Psi_{\epsilon}},
\end{aligned}
$$

leading to

$$
\left\|(T u)^{\prime}\right\|_{k} \leq\left(f^{\infty}+\epsilon\right) \Gamma_{2}\|u\|+\overline{\Psi_{\epsilon}} .
$$

Summing (17) with (18), we obtain

$$
\|T u\| \leq\left(f^{\infty}+\epsilon\right) \Gamma^{-1}\|u\|+2 \overline{\Psi_{\epsilon}} \leq\|u\| .
$$

We deduce from Assertion 2 of Theorem 2, that $T$ admits a fixed point $u \in P$ with $R_{1} \leq\|u\| \leq R_{2}$ which is, by Lemma 3, a positive solution to Problem (5).

Thus, the proof of Theorem 1 is complete.

\section{Comments}

1. Notice that the obtained positive solution in Theorem 1 is nondecreasing and bounded. Indeed, if $u \in P \backslash\{0\}$ is a fixed point of $T$ with $\|u\|=R$, then for all $t>0$

$$
u^{\prime}(t)=(T u)^{\prime}(t)=\int_{0}^{+\infty} \widetilde{G}(t, s) \phi(s) f\left(s, u(s), u^{\prime}(s)\right) d s>0
$$

and Hypothesis (6) leads to

$$
u(t)=T u(t)=\int_{0}^{+\infty} G(t, s) \phi(s) f\left(s, u(s), u^{\prime}(s)\right) d s
$$




$$
\begin{aligned}
& \leq \int_{0}^{+\infty} \mathrm{G}(\mathrm{t}, \mathrm{s}) \phi(\mathrm{s}) \Psi_{\mathrm{R}}\left(\left(\mathrm{e}^{-\mathrm{ks}} \mathrm{u}(\mathrm{s})\right),\left(\mathrm{e}^{-\mathrm{ks}} \mathrm{u}^{\prime}(\mathrm{s})\right)\right) \mathrm{ds} \\
& \leq \frac{1}{\mathrm{k}^{2}} \int_{0}^{+\infty} \phi(\mathrm{s}) \Psi_{\mathrm{R}}\left(\operatorname{Re}^{-\mathrm{k} s} \gamma(\mathrm{s}), \operatorname{Re}^{-\mathrm{ks}} \widetilde{\gamma}(\mathrm{s})\right) \mathrm{d} s<\infty .
\end{aligned}
$$

2. From the above comment arise the following question. Why we looked for solutions in the space $E$ instead of looking for them in the natural space

$\mathrm{F}=\left\{\mathrm{u} \in \mathrm{C}^{1}\left(\mathbb{R}^{+}\right): \max \left(\sup _{\mathrm{t}>0}|\mathrm{u}(\mathrm{t})|, \sup _{\mathrm{t}>0}\left|\mathrm{u}^{\prime}(\mathrm{t})\right|\right)<\infty\right\} ?$

The answer is: There is no cone in $\mathrm{F}$ where we can realize the inequality $\|\mathrm{Tu}\| \geq\|\mathrm{u}\|$ in Theorem 2 .

3. Notice that for $\theta>1, \Gamma<\Theta_{0}(\theta)<\Theta_{\infty}(\theta)$ and let the interval $\mathcal{I}=$ $\left(\Gamma, \Theta_{\infty}(\theta)\right)$. In the particular case where the limits

$$
f^{0}=\lim _{|(w, z)| \rightarrow 0} \frac{f\left(t, e^{k t} w, e^{k t} z\right)}{w+z}, \quad f^{\infty}=\lim _{|(w, z)| \rightarrow 0} \frac{f\left(t, e^{k t} w, e^{k t} z\right)}{w+z}
$$

exist, then Theorem 1 claims that the bvp (5) admits a positive solution if $f^{0}$ and $f^{\infty}$ are oppositely located relatively to the interval $\mathcal{I}$, that is the ratio $\left(\mathrm{f}\left(\mathrm{t}, \mathrm{e}^{\mathrm{kt}} w, e^{\mathrm{kt}} z\right) / w+z\right)$ crosses the interval $\mathcal{I}$. Two questions arise from this observation; what happens if $\left(\mathrm{f}\left(\mathrm{t}, e^{\mathrm{kt}} w, e^{\mathrm{kt}} z\right) / w+z\right)>\Theta_{\infty}(\theta)$ or $\left(\mathrm{f}\left(\mathrm{t}, \mathrm{e}^{\mathrm{kt}} \mathcal{w}, e^{\mathrm{kt}} z\right) / w+z\right)<\Gamma$ for all $\mathrm{t}, w, z>0$ ?

The second question is: are the constants $\Gamma, \Theta_{0}(\theta), \Theta_{\infty}(\theta)$ the best ones? In an other manner, does exist two positive constants $\alpha$ and $\beta$ with $\Gamma<$ $\alpha<\beta<\Theta_{0}(\theta)$ such that if $f^{0}$ and $f^{\infty}$ are oppositely located relatively to the interval $(\alpha, \beta)$, then the bvp (5) admits a positive solution?

4. Let $p>1$ and consider the case where $E$ is equipped with the norm $\|u\|_{p}=\sqrt[p]{\|u\|_{k}^{p}+\|u\|_{k}^{p}}$. In this case, under Hypothesis (6), we prove by the same arguments that the bvp (5) admits a positive solution if $f^{0}<\Gamma_{p}<\Theta_{\infty}^{p}(\theta)<f_{\infty}(\theta)$ for some $\theta>1$ or $f^{\infty}<\Gamma<\Theta_{0}^{p}(\theta)<f_{0}(\theta)$ for some $\theta>0$, where

$$
\begin{aligned}
& \Gamma_{p}=\left(\left(\Gamma_{1}\right)^{p}+\left(\Gamma_{2}\right)^{p}\right)^{-1 / p}, \\
& \Theta_{0}^{p}(\theta)=\left(\left(\Theta_{1,0}(\theta)\right)^{p}+\left(\Theta_{2,0}(\theta)\right)^{p}\right)^{-1 / p} \text { for } \theta>0, \\
& \Theta_{\infty}^{p}(\theta)=\left(\left(\Theta_{1, \infty}(\theta)\right)^{p}+\left(\Theta_{2, \infty}(\theta)\right)^{p}\right)^{-1 / p} \text { for } \theta>0 .
\end{aligned}
$$

Noticing that $\Gamma_{p}>\Gamma, \Theta_{0}^{p}(\theta)>\Theta_{0}(\theta)$ and $\Theta_{\infty}^{p}(\theta)>\Theta_{p, \infty}(\theta)$ we understand that the problem posed in the above comment is a serious problem. 


\section{Acknowledgement}

The authors are thankful to the anonymous referee for his careful reading of the manuscript and for all his comments and suggestions, which led to a substantial improvement of the original manuscript.

\section{References}

[1] R. P. Agarwal and D. O'Regan, Infinite interval problems for differential, difference and integral equations, Kluwer Academic Publisher, Dordrecht, 2001.

[2] C. Bai and C. Li, Unbounded upper and lower solution method for thirdorder boundary value problem on the half-line, Electron. J. Differential Equations, 2009 No. 119 (2009), 1-12.

[3] Z. Benbaaziz and S. Djebali, On a singular multi-point third-order boundary value problem on the half-line, Mathematica Bohemica, (2019), DOI: 10.21136/MB.2019.0084-18.

[4] F. Bernis and L. A. Petelier,Two problems from draining flows involving third-order ordinary differential equations, SIAM J. Math. Anal., 27 No. 2 (1996), 515-527.

[5] C. Corduneanu, Integral Equations and Stability of Feedback Systems, Academic Press, New York, 1973.

[6] S. Djebali and O. Saifi, Singular $\phi$-Laplacian third-order BVPs with derivative dependence, Arch. Math. (Brno), 52 (2016), 35-48.

[7] S. Djebali and O. Saifi, Upper and lower solution for $\phi$-Laplacian thirdorder BVPs on the half-line, Cubo A Mathematical Journal, 16 No. 1 (2014), 105-116.

[8] S. Djebali and O. Saifi, Third order BVPs with $\phi$-Laplacian operators on $[0,+\infty)$, Afr. Diaspora J. Math., 16 No. 1 (2013), 1-17.

[9] S. Djebali and O. Saifi, Positive solutions for singular BVPs with sign changing and derivative depending nonlinearity on the half-line, Acta Appl. Math., 110 (2010), 639-665. 
[10] X. Feng, H. Feng and H. Tan, Existence and iteration of positive solutions for third-order Sturm-Liouville boundary value problem, Appl. Math. Comput., 266 (2015), 634-641.

[11] Y. Feng, On the existence and multiplicity of positive periodic solutions of a nonlinear third-order equation, Appl. Math. Lett., 22 (2009), 1220-1224.

[12] D. Fu and W. Ding, Existence of positive solutions for third-order boundary value problem with integral boundary conditions in Banach spaces, Adv. Difference Equ., 2013, 2013:65.

[13] J. R. Graef, L. Kong and B. Yong, Positive solutions for third-order multipoint singular boundary value problems, Czechoslovak Math. J., 60 No. 135 (2010), 173-182.

[14] Y. Guo, Y. Liu and Y. Liang, Positive solutions for the third-order boundary value problems with the second derivatives, Bound. Value Probl., 2012, 2012:34.

[15] N. Finizio and G. Ladas, Ordinary Differential Equations with Modern Applications, Third Edition, Wadsworth Pub. Co., Belmont, 1988.

[16] S.[A. Iyase, On a third-order three point boundary value problem at resonance on the half-line, Arab. J. Math. (2019), 8:43-53.

[17] D. Krajcinovic, Sandwich Beam Analysis, Appl. Mech., 1 No. 39, (1972), 773-778.

[18] Y. Kuramoto and T. Yamada, Turbulent state in chemical reaction, Progress of Theoretical Physics, 56 (1976), 679.

[19] H. Lian and J. Zhao, Existence of unbounded solution for a third-order boundary value problem on infinite intervals, Discrete Dyn. Nat. Soc., Vol. 2012, Article ID 357697, 14 pages, doi:101155/2012/

[20] S. Liang and J. Zhang, Positive solutions for singular third-order boundary-value problem with dependence on the first order derivative on the half-line, Acta. Appl. Math., 111 (2010), 27-43.

[21] Z. Liu, H. Chen and C. Liu, Positive solutions for singular third-order nonhomogeneous boundary value problems, J. Appl. Math. Comput., 38 (2012), 161-172. 
[22] H. P. McKean, Nagumo's equation, Advances in Mathematics, 4 (1970), 209-223.

[23] D. Michelson, Steady solutions of the Kuramoto-Sivashinsky equation, Physica D, 19 (1986), 89-111.

[24] P. K. Palamides and R. P. Agarwal, An existence result for a singular third-order boundary value problem on $[0,+\infty)$, Appl. Math. Lett., 21 (2008), 1254-1259.

[25] H. Pang, W. Xie and L. Cao, Successive iteration and positive solutions for a third-order boundary value problem involving integral conditions, Bound. Value Probl., (2015), 2015:39.

[26] H. Shi, M. Pei and L. Wang, Solvability of a third-order three point boundary value problem on a half-line, Bull. Malays. Math. Sci. Soc., 38 No. 3 (2015), 909-926.

[27] Y. Sun, Triple positive solutions for a class of third-order p-Laplacian singular boundary value problems, J. Appl. Math. Comput., 37 (2011), $587-599$.

[28] Z. Wei, Some necessary and sufficient conditions for existence of positive solutions for third-order singular sublinear multi-point boundary value problems, Acta Math. Sin., 34 B (6) (2014), 1795-1810.

[29] Z. Wei, Some necessary and sufficient conditions for existence of positive solutions for third-order singular super-linear multi-point boundary value problems, J. Appl. Math. Comput., 46 (2014), 407-422.

[30] Y. Wu and Z. Zhao, Positive solutions for a third-order boundary value problems with change of signs, Appl. Math. Comput., 218 (2011), 27442749 .

[31] J. Zhang, Z. Wei and W. Dong, The method of lower and upper solutions for third-order singular four-point boundary value problems, $J$. Appl. Math. Comput., 36 (2011), 275-289. 